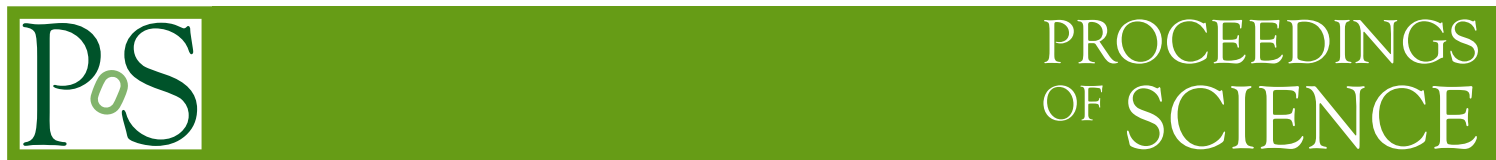

\title{
Light scalar dark matter at neutrino oscillation experiments
}

\author{
Kerry Whisnant ${ }^{* \dagger}$ \\ Iowa State University \\ E-mail: whisnanteiastate.edu
}

\begin{abstract}
Couplings between light scalar (fuzzy) dark matter (DM) and neutrinos induce a perturbation to the neutrino mass matrix. If the DM oscillation period is smaller than ten minutes (or equivalently, the DM particle is heavier than $0.69 \times 10^{-17} \mathrm{eV}$ ), the fast-averaging over an oscillation cycle leads to a modification of the measured oscillation parameters. We present a specific $\mu$ - $\tau$ symmetric model in which the measured value of $\theta_{13}$ is entirely generated by the DM interaction, and which reproduces the other measured oscillation parameters. For a scalar DM particle lighter than $10^{-15}$ $\mathrm{eV}$, adiabatic solar neutrino propagation is maintained. A suppression of the sensitivity to $C P$ violation at long baseline neutrino experiments is predicted in this model. We find that DUNE cannot exclude the DM scenario at more than $3 \sigma$ C.L. for bimaximal, tribimaximal and hexagonal mixing, while JUNO can rule it out at more than $6 \sigma$ C.L. by precisely measuring both $\theta_{12}$ and $\theta_{13}$.
\end{abstract}

The 20th International Workshop on Neutrinos (NuFact2018)

12-18 August 2018

Blacksburg, Virginia

\footnotetext{
${ }^{*}$ Speaker.

${ }^{\dagger}$ in collaboration with J. Liao and D. Marfatia
} 


\section{Introduction}

Fuzzy DM [1] with a mass range $1-10 \times 10^{-22} \mathrm{eV}$ has attracted much attention recently since it can resolve the small scale crisis for standard cold DM due to its large de Broglie wavelength [2]. Constraints on fuzzy DM can be obtained from Lyman- $\alpha$ forest data and a lower limit of $20 \times$ $10^{-22} \mathrm{eV}$ at $2 \sigma$ C.L. has been set from a combination of XQ-100 and HIRES/MIKE data [3], although a proper handling of the effect of quantum pressure and systematic uncertainties may relax the limit [4]. A popular production mechanism for generating light scalar DM is the misalignment mechanism, in which the DM field starts to oscillate as a coherent state with a single macroscopic wavefunction [5].

The properties of light scalar DM can be probed if they are coupled to Standard Model (SM) fermions, which induce a time variation to the masses of the SM fermions due to the oscillation of the DM field. Here we consider the couplings between the light scalar DM and the SM neutrinos, which were first studied in Ref. [6]; constraints on light scalar DM couplings were also considered in Refs. [7, 8]. In general, the interactions between DM and neutrinos provide a small perturbation to the neutrino mass matrix. If the DM oscillation period is much smaller than the periodicity to which an experiment is sensitive, the oscillation probabilities get averaged, and a modification of the oscillation parameters can be induced if the data are interpreted in the standard three-neutrino framework. It is this kind of scenario that we consider in this talk.

\section{The model}

The Lagrangian describing the interactions between light scalar DM and neutrinos can be written in the flavor basis as [7, 8] $\mathscr{L}=\bar{v}_{L \alpha} i \not \partial v_{L \alpha}-\frac{1}{2} M_{0}^{\alpha \beta} \overline{v_{L \alpha}^{c}} v_{L \beta}-\frac{1}{2} \lambda \alpha \beta \phi \overline{v_{L \alpha}^{c}} v_{L \beta}+$ h.c., where $\alpha, \beta=e, \mu, \tau, M_{0}$ is the initial neutrino mass matrix, and $\lambda$ is the coupling constant matrix. Since the light scalar DM can be treated as a classical field, the nonrelativistic solution to the classical equation of motion can be approximated [6] as $\phi(x) \simeq \frac{\sqrt{2 \rho_{\phi}(x)}}{m_{\phi}} \cos \left(m_{\phi} t\right)$, where $m_{\phi}$ is the mass of the scalar DM particle, $\rho_{\phi} \sim 0.3 \mathrm{GeV} / \mathrm{cm}^{3}$ is the local DM density, and a term in the cosine argument involving the negigible virialized DM velocity has been neglected.

The effective mass matrix can be treated as the sum of an initial mass matrix and a small perturbation [9], i.e., $M=U_{0}^{*} M_{D} U_{0}^{\dagger}+\mathscr{E} \cos \left(m_{\phi} t\right)$, where $U_{0}$ is the initial mixing matrix, $M_{D}$ is the mass matrix in the mass basis (with $m_{i}^{0}$ as the initial neutrino eigenmasses), and the elements of the perturbation matrix are $\mathscr{E} \alpha \beta=\lambda^{\alpha \beta} \sqrt{2 \rho_{\phi}} / m_{\phi}$.

We considered [10] a $\mu$ - $\tau$ symmetric model (with initial mixing angles $\theta_{23}=\pi / 4$ and $\theta_{13}^{0}=0$ ) in which the DM masses do not affect the neutrino masses at leading order in the perturbation; such a model has a perturbation of the form

$$
\mathscr{E}=\left(\begin{array}{ccc}
0 & \varepsilon & \varepsilon \\
\varepsilon & \varepsilon^{\prime} & 0 \\
\varepsilon & 0 & -\varepsilon^{\prime}
\end{array}\right) .
$$

From the generalized perturbation results of Ref. [9], the leading order corrections to the mixing angles have amplitudes $\delta \theta_{13} \approx \sqrt{2}|\varepsilon| / \delta m_{31}, \delta \theta_{23} \approx \operatorname{Re}\left(\varepsilon^{\prime}\right) / \delta m_{31}, \delta \theta_{12} \approx-\operatorname{Re}\left[\sqrt{2} \varepsilon \varepsilon^{\prime} \cos 2 \theta_{12}^{0}+\right.$ $\left.\left(\varepsilon^{2}-\varepsilon^{\prime 2} / 2\right) \sin 2 \theta_{12}^{0}\right] /\left(\delta m_{21} \delta m_{31}\right)$, and $\delta_{C P} \approx \arg (\varepsilon)$. Note that $\delta \theta_{12}$ is proportional to the product 
of two $\varepsilon$ 's and therefore has a DM oscillation factor $\cos ^{2}\left(m_{\phi} t\right), \delta \theta_{13}$ and $\delta \theta_{23}$ are linearly dependent on $\cos \left(m_{\phi} t\right)$, while $\delta_{C P}$ does not depend on the DM oscillation because it is equal to the phase of $\varepsilon$ and does not depend on the magnitude.

\section{Phenomenology}

Using the superscript ' 0 ' to denote the initial oscillation parameters before the DM perturbation, the effective parameters that are measured in reactor, solar, and atmospheric experiments are $\theta_{23}^{e f f}=\theta_{23}^{0}, \theta_{12}^{e f f}=\theta_{12}^{0}+\frac{1}{2} \delta \theta_{12}$, and $\theta_{13}^{e f f}=\delta \theta_{13} / \sqrt{2}$, after averaging over the DM oscillation. Note that $\theta_{23}$ remains maximal at leading order and that the unperturbed $\theta_{12}^{0}$ need not be near the experimentally measured value. We considered three possibilities: $\theta_{12}^{0}=45^{\circ}$ for bimaximal (BM) mixing, $\theta_{12}^{0}=35.3^{\circ}$ for tri-bimaximal (TBM) mixing, and $\theta_{12}^{0}=30^{\circ}$ for hexagonal (HG) mixing.

Since the currently running long-baseline experiments, T2K and NOvA, have large experimental uncertainties, we considered the next-generation DUNE program. We found [10] that DUNE alone cannot distinguish the DM scenario from the SM scenario at more than the $3 \sigma$ C.L. if $m_{1}$ is greater than about $0.05 \mathrm{eV}$. For a smaller $m_{1}$, the magnitude of $\varepsilon$ required to explain the measured $\theta_{13}$ becomes larger, and higher order corrections then break the degeneracies between the SM and DM scenarios.

Since future medium-baseline reactor experiments can make a high precision measurement of both $\theta_{12}$ and $\theta_{13}$, we also studied [10] the sensitivity reach at JUNO. For the lightest mass between 0 and $0.2 \mathrm{eV}$, we found that the minimum value of $\chi^{2}$ at JUNO is $47.6,46.9$ and 57.0, with the initial mixing being BM, TBM and HG, respectively. Hence, JUNO can rule out this model with those initial mixings at more than $6 \sigma$ C.L.

\section{References}

[1] W. Hu, R. Barkana and A. Gruzinov, Phys. Rev. Lett. 85, 1158 (2000) [astro-ph/0003365].

[2] L. Hui, J. P. Ostriker, S. Tremaine and E. Witten, Phys. Rev. D 95, no. 4, 043541 (2017) [arXiv:1610.08297 [astro-ph.CO]].

[3] V. Irsic, M. Viel, M. G. Haehnelt, J. S. Bolton and G. D. Becker, Phys. Rev. Lett. 119, no. 3, 031302 (2017) [arXiv:1703.04683 [astro-ph.CO]].

[4] J. Zhang, J. L. Kuo, H. Liu, Y. L. S. Tsai, K. Cheung and M. C. Chu, arXiv:1708.04389 [astro-ph.CO].

[5] A. Suarez, V. H. Robles and T. Matos, Astrophys. Space Sci. Proc. 38, 107 (2014) [arXiv:1302.0903 [astro-ph.CO]].

[6] A. Berlin, Phys. Rev. Lett. 117, no. 23, 231801 (2016) [arXiv:1608.01307 [hep-ph]].

[7] G. Krnjaic, P. A. N. Machado and L. Necib, arXiv:1705.06740 [hep-ph].

[8] V. Brdar, J. Kopp, J. Liu, P. Prass and X. P. Wang, Phys. Rev. D 97, no. 4, 043001 (2018) [arXiv:1705.09455 [hep-ph]].

[9] A generic treatment of small perturbations on the neutrino mass matrix is provided in J. Liao, D. Marfatia and K. Whisnant, Phys. Rev. D 87, no. 1, 013003 (2013) [arXiv:1205.6860 [hep-ph]]; Phys. Rev. D 92, no. 7, 073004 (2015) [arXiv:1506.03013 [hep-ph]].

[10] J. Liao, D. Marfatia and K. Whisnant, JHEP 1804, 136 (2018) [arXiv:1803.01773 [hep-ph]]. 